\title{
Diavoli, bombe atomiche e mass media. Il punto di vista di Pasolini e Moravia
}

Chiara Lombardi

\section{OpenEdition}

Journals

Edizione digitale

URL: http://journals.openedition.org/cei/101

DOI: $10.4000 /$ cei. 101

ISSN: 2260-779X

Editore

UGA Éditions/Université Grenoble Alpes

\section{Edizione cartacea}

Data di pubblicazione: 15 juin 2010

Paginazione: $41-51$

ISBN: 978-2-84310-168-7

ISSN: 1770-9571

Notizia bibliografica digitale

Chiara Lombardi, «Diavoli, bombe atomiche e mass media. II punto di vista di Pasolini e Moravia»,

Cahiers d'études italiennes [Online], 11 | 2010, online dal 15 décembre 2011, consultato il 26 mars 2021.

URL: http://journals.openedition.org/cei/101 ; DOI: https://doi.org/10.4000/cei.101 


\title{
DIAVOLI, BOMBE ATOMICHE E MASS MEDIA. IL PUNTO DI VISTA DI PASOLINI E MORAVIA
}

\author{
Chiara Lombardi \\ Université de Turin
}

Senza mezzi termini, né falsi pudori e nemmeno paure di essere «inattuale», nelle Lettere luterane Pasolini afferma di voler comporre un «trattato pedagogico». Nei primi capitoli di questa raccolta - dedicata al personaggio di Gennariello, da intendersi come una sorta di lettore implicito o lettore ideale - lo scrittore prende perciò in considerazione le "fonti educative» più immediate: dal «linguaggio pedagogico» delle cose, «mute, materiali, oggettuali, inerti, puramente presenti» (come le merci e i beni di consumo), a quello trasmesso dalla famiglia e dai genitori, «educatori ufficiali» anche se talvolta «diseducatori», a quello della scuola e della stessa «antiscuola» - la polemica politica contro la scuola, che impone un conformismo altrettanto angosciante. Nell'ultima parte del volume lo scrittore non trascura di prendere in considerazione anche i media: "la stampa e la televisione», che definisce "questi spaventosi organi pedagogici privi di alcuna alternativa» (LL, pp. 3I-33).

In questa analisi che Pasolini rivolge ai mezzi di comunicazione e alle sue forme, ai canali privilegiati di trasmissione della contemporaneità con cui un ragazzo si trova a confrontarsi nell'Italia degli anni Settanta, è evidentemente centrale il linguaggio - segno e sintomo della vitalità di una cultura come del suo soffocamento. Si pensi alle acutissime osservazioni sul linguaggio degli slogan in Scritti corsari (dall'articolo apparso sul Corriere della sera, "Il folle slogan dei Jeans Jesus»), in cui si mette in evidenza non solo la forma irrigidita e stereotipata dello stesso slogan, «il contrario dell' espressività, che è eternamente cangiante» (SC, p. I4), ma anche la rivelazione di sé come segno duplice: nuovo valore, laico ma blasfemo, dell' «entropia borghese» che usa la religione come propulsore di enorme consumo e, al tempo stesso, pretesto per considerare la ridicola reazione della stampa religiosa (si allude a un articolo dell'Osservatore 
romano): «con il suo italianoccio antiquato, spiritualistico e un po' fatuo, l'articolista dell' Osservatore intona un treno, non certo biblico, per fare del vittimismo da povero, indifeso innocente. È lo stesso tono con cui sono redatte, per esempio, le lamentazioni contro la dilagante immoralità della letteratura o del cinema» (SC, p. I5). La Chiesa (e i giornalisti che scrivono per tale istituzione), insomma, colpiscono laddove e nei termini in cui non dovrebbero colpire, senza comprendere come non si tratti di una semplice blasfemia, ma di quella espressione - fenomeno ormai irreversibile per la società italiana - del Neocapitalismo quale unico dio della borghesia.

Oltre alle considerazioni che dedica al linguaggio del potere consumistico, nelle Lettere luterane Pasolini esprime la propria indignata reazione alla parola dei politici, dei democristiani innanzitutto, tra i quali ravvisa «i diretti responsabili o mandanti della strategia della tensione e delle bombe». Di queste stragi essi non parlano, mentre si esprimono laddove non dovrebbero parlare:

non solo restano al potere, ma parlano. Ora è la loro lingua che è la pietra dello scandalo. Infatti ogni volta che aprono bocca, essi, per insincerità, per consapevolezza, per paura, per furberia, non fanno altro che mentire. La loro lingua è la lingua della menzogna. E poiché la loro cultura è una putrefatta cultura forense e accademica, mostruosamente mescolata con la cultura tecnologica, in concreto la loro lingua è pura teratologia. Non si può ascoltare. Bisogna tapparsi le orecchie. (LL, p. 29.)

In Scritti corsari, da un articolo uscito per il Corriere della sera («Il romanzo delle stragi», I4 novembre 1974), lo scrittore ribadisce:

i politici e i giornalisti, pur avendo forse delle prove e certamente degli indizi, non fanno i nomi. [...] Il potere e il mondo che, pur non essendo del potere, tiene rapporti pratici con il potere, ha escluso gli intellettuali liberi - proprio per il modo in cui è fatto - dalla possibilità di avere prove e indizi. (SC, p. 75.)

Quale soluzione, allora, di fronte a questa forma di mistificazione linguistica o di implicita censura operata all'interno della democrazia stessa? Nelle Lettere si mette in evidenza come il compito dell'educatore, comunque, sarebbe di insegnare a non ascoltare tali «mostruosità linguistiche». «In altre parole», precisa Pasolini, «il dovere degli intellettuali sarebbe quello di rintuzzare tutte le menzogne che attraverso la stampa e soprattutto la televisione inondano e soffocano quel corpo del resto inerte che è l'Italia» (LL, p. 30).

Tale è il motivo - ridare coscienza e vigore a quel «corpo», inerte, ma sempre da intendersi come tale, come composto di uomini fatti di parola e fisicità - per cui Pasolini non si rassegna a tacere, ma sceglie di smuovere 
questo acritico, atrofizzato ristagno intellettuale e corporale sia attraverso la poesia, ad esempio con voce friulana o romanesca (qui dichiara che vorrebbe parlare napoletano, se non fosse capace), sia nel cinema (si veda la Trilogia della vita, con Le mille e una notte, il Decamerone e i Racconti di Canterbury, di cui parla nelle stesse Lettere), sia attraverso gli stessi media di cui inevitabilmente fa parte, senza risparmiarsi davvero di sprofondare all'interno di questi canali di comunicazione, come dimostra negli articoli per Il Corriere e Il Mondo pubblicati negli Scritti corsari. Un empito quasi biblico, un ferire di spada che di spada ferisce, ma anche una dichiarazione d'amore che esplode proprio nel momento della sua negazione (nel momento stesso in cui dichiara, verso i giovani, il suo disamore e la sua condanna, LL, p. 6). Un inno all' «Ombra sdegnosa» di De Sade piuttosto che all' «ombra mostruosa di Rousseau», le Lettere Luterane, scritto con la speranza che il libro non sia semplicemente preso tra i testi «leggibili» (LL, p. 33).

Particolarmente importante mi sembra questa allusione a Roland Barthes, al «bellissimo libro» Il piacere del testo (LL, p. 33), che si coglie non soltanto attraverso l'implicita distinzione tra testi «leggibili» - accondiscendenti, colti, piacevoli e confortevoli, ma non realmente innovativi né deflagranti - e testi «scrivibili», testi di godimento, in cui prorompe la jouissance e si dispiega la "natura asociale del piacere» (Barthes, 1999, pp. 85 e I04). Credo che ogni aspetto di questa scrivibilità del testo affermata da Barthes valga poi implicitamente per Pasolini come norma (l'unica, la più efficace) capace di contrastare quei meccanismi di appiattimento della cultura, dell'intelligenza, della corporeità stessa coinvolta nell'atto intellettuale generati dalla più banale comunicazione massmediologica. Il testo di godimento, per Barthes (e nella sua stessa citazione di De Sade), è quello che deriva da "certe rotture (o da certe) collisioni», ad esempio quelle tra "codici antipatici» che entrano in contatto (Barthes, I999, p. 77), quello della perdita e della distruzione di quell'immaginario e di quel piacere strumentalizzato per creare delle condivisioni o delle gerarchie ideologiche (cfr. Žižek, 2006, pp. 139-I69). Se il linguaggio capitalista è «implacabile invischiamento», doxa, ideologia, infatti, il testo letterario è quello del paradosso, dell'atopia, dell'indicibile, che fa a pezzi il piacere stesso, appunto, e con esso la lingua e la cultura, che «mostra il didietro al Potere Politico» (Barthes, 1999, p. II5). È il testo che rivive come corpo, "corpo anonimo dell'attore dentro il mio orecchio: qualcosa granula, crepita, accarezza, raspa, taglia: gioisce» (Barthes, 1999, p. 127).

Credo che sia attraverso questo immediato entusiasmo per la critica bartiana che vadano comprese molte prese di posizione, implicite (quindi 
letterarie) ed esplicite (giornalistiche) di Pasolini (su questa distinzione che può apparire un po' vaga tornerò a parlare) rispetto alla comunicazione dei nuovi media, che andava e sarebbe andata (e lo dimostra il mondo di oggi, degli ultimi trent'anni) sempre più in direzione contraria rispetto a quella stessa forza del testo rivendicata da Barthes. Non si tratta soltanto, per Pasolini, di un problema letterario o critico. Per lo scrittore l'atteggiamento dei media è tra i primi responsabili della criminalità e del suo legame con le trasformazioni sociali dell'Italia. La televisione e la scuola media dell'obbligo sono provocatoriamente eliminate dal progetto educativo delle Lettere Luterane, come è spiegato in Due proposte per eliminare la criminalità in Italia, in quanto principali responsabili di un'educazione di massa sempre più tendente alla criminalità. La scuola media dell'obbligo e la televisione danno l'illusione di un falso progresso della cultura (il sottotitolo delle Lettere è proprio Il progresso come falso progresso) e servono all' «iniziazione alla qualità di vita piccolo borghese» (LL, p. I69). Sono, quindi, strumenti della mentalità piccolo borghese: non poca cultura perché è improduttiva, non troppa perché è scomoda. Quel tanto che basta a sviluppare una massa utile a soddisfare le esigenze della società italiana e ad aderire alla sua classe politica dominante senza dare troppo fastidio, insomma. È questa la «retorica progressista» della democrazia (cristiana) degli anni Sessanta e Settanta. A differenza della scuola, inoltre, la televisione gioca un ruolo ancora più incisivo, sotto certi aspetti, in quanto «esempio», i cui «modelli» non vengono parlati, ma rappresentati.

E se i modelli son quelli, come si può pretendere che la gioventù più esposta e indifesa non sia criminaloide o criminale? E stata la televisione che ha, praticamente (essa non è che un mezzo) concluso l'era della pietà, e iniziato l'era dell'edonè. Era in cui i giovani insieme presuntuosi e frustrati a causa della stupidità e insieme dell'irraggiungibilità dei modelli proposti loro dalla scuola e dalla televisione, tendono inarrestabilmente ad essere aggressivi fino alla delinquenza o passivi fino all'infelicità (che non è una colpa minore). (LL, p. I70.)

Non si tratta soltanto di una forma di emulazione, ma di una connivenza nella degenerazione di certi meccanismi sociali. È molto interessante vedere come il concetto dell' «era dell'edoné» sia poi ripreso, in ambito sociologico, dal filosofo Slavoj Žižek, il quale ha scritto che «tutta la politica poggia su un certo livello di economica del godimento, che essa peraltro manipola» (Žižek, 2006, p. I43), e ha sviluppato questo concetto in gran parte dei suoi scritti (tra gli altri, cfr. Žižek, 200I, 2003, 2008).

Per Pasolini, uno degli esempi più significativi di questa emulazione popolare dei modelli televisivi, mediativi e del loro potere di manipo- 
lazione è rappresentato dal massacro del Circeo. Nell'ambito della «solita ondata di stupidità giornalistica» (LL, p. I66), l'episodio dà modo allo scrittore di confrontarsi con altri intellettuali italiani come Calvino (il quale si era espresso in un articolo del Corriere della Sera dell'8 ottobre 1975) e, appunto, Moravia, con i quali si trova a dissentire. In Lettera luterana a Italo Calvino, lo scrittore isola un'analisi - quella calviniana - perfettamente illuminante: la carneficina come qualcosa di "perfettamente naturale», la "permissività assoluta» che ha incoraggiato quel gesto; l'estendersi nella nostra società di «stati cancerosi»; l'atonia morale e l'irresponsabilità sociale, etc. (LL, pp. I79-I84). Secondo Pasolini la critica di Calvino non fa che incoraggiare un luogo comune trasmesso anche dai media, ossia che i responsabili sono dei neofascisti, i quali hanno trovato terreno fertile in quella atonia sociale, in quello stesso clima di permissività assoluta di cui si è detto, e così via:

Tu hai privilegiato i neofascisti pariolini del tuo interesse e della tua indignazione, perché sono borghesi. La loro criminalità ti pare interessante perché sono borghesi. La loro criminalità ti pare interessante perché riguarda i nuovi figli della borghesia. Li porti dal buio truculento della cronaca alla luce dell'interpretazione intellettuale, perché la loro classe sociale lo pretende. Ti sei comportato - mi sembra - come tutta la stampa italiana, che negli assassini del Circeo vede un caso che la riguarda, un caso, ripeto, privilegiato. (p. 182)

Aldilà della riflessione - mai troppo scontata - che la televisione rappresenti un modello efficacissimo di emulazione proprio attraverso i suoi meccanismi di fruizione e di rappresentazione, mi sembra che Pasolini abbia colto l'aspetto più sottile e tragico di questo episodio che (come l'attuale delitto di Cogne) si pone al centro dell'esperienza mediatica: l'interesse per la classe sociale in cui sono coinvolti i protagonisti della vicenda, "un caso che la riguarda", che coinvolge più da vicino la stampa e la società «bene» dell'Italia. Si tratta non solo di non comprendere questo meccanismo sociale, ma di incoraggiarlo. Interessarsi a un delitto perché compiuto in seno alla borghesia più che a un delitto tra borgatari e immigrati, individuare come capro espiatorio un male che deresponsabilizza (il neofascismo) vuole dire isolare come fenomeno di interesse un episodio descritto come proprio di una classe soggetta ad emulazione, quindi indirettamente farne un genere letterario. $\mathrm{E}$, in fondo, la teoria aristotelica del protagonista della tragedia come chrestos, l'uomo nobile e ricco, e dell'esclusione del phaulos, l'uomo «dappoco» riservato allo stile comico. Ora essa si applica non al linguaggio sublime della tragedia stessa, ma al contagioso «verbalismo» di molti giornalisti, «complici degli uomini politici» (LL, p. I57) ed emuli a loro volta del linguaggio della politica, di 
cui rispecchiano la «caotica quotidianità»e la restituiscono in forma pericolosamente «mitizzata». Così avviene per il caso dei Circeo, che diventa - proprio attraverso la risonanza mediatica e il conformismo di certi intellettuali - un "genere di consumo». Se infatti i proletari e i sottoproletari italiani sono divorati dall' "ansia economica», non aspettano altro che di uniformarsi alla massa di piccolo borghesi, nell'emulazione di qualsiasi loro atto - e specie di quelli che salgono alla ribalta dei mass media.

Gli allettamenti del consumismo, la falsa retorica progressista hanno trasformato il «mondo reale» in «una totale irrealtà, dove non c'è più scelta possibile tra male e bene» (un fenomeno che, non a caso, è stato attribuito anche ad Arancia meccanica, di poco precedente e letto come segno di incapacità di distinguere tra bene e male). La falsa tolleranza soffoca le emozioni, le stempera, a vantaggio della superba indifferenza borghese, della riduzione dell'uomo a mezzo.

Sono concetti che attraversano anche la riflessione di Alberto Moravia, soprattutto nel saggio L'uomo come fine, dove lo scrittore individua nella società del Novecento "lo scadimento dell'umanesimo tradizionale; la sua immobilità, il suo conservatorismo; la sua ipocrisia di fronte agli eventi tragici della prima metà del secolo»:

L'uomo del neocapitalismo con tutti i suoi frigoriferi, i suoi supermarket, le sue automobili utilitarie, i suoi missili e i suoi set televisivi è tanto esangue, sfiduciato, devitalizzato e nevrotico da giustificare coloro che vorrebbero accettarne lo scadimento quasi fosse un fatto positivo e ridurlo a oggetto tra gli oggetti [...] Sotto apparenza scintillanti e astratte, si celano, a ben guardare, la noia, il disgusto, l'impotenza, l'irrealtà. (UF, pp. 3-4.)

Sia Moravia sia Pasolini rivolgono la loro attenzione a quel mondo divenuto irreale, astratto, espressione di un antiumanesimo che ha privato l'uomo non di una presuntuosa centralità, ma che lo ha ridotto a mezzo, a «oggetto tra gli oggetti», interprete disgustato di una corsa cieca che ha il suo movimento e il suo approdo nella perdita del rispetto per il linguaggio più vicino alle cose e alle emozioni, per la corporeità propria e altrui e nella incapacità di riconoscere, di esaltare e, insieme, di contenere le proprie passioni fuori dal potere economico e da quello della ribalta televisiva e, in genere, mediatica. Penso che, in questo senso, il prezioso punto di vista di Pasolini e di Moravia ci aiutino anche a capire le dinamiche che reggono le attuali e quotidiane offerte di delitti e di massacri, nella loro cieca esecuzione e nella stessa risonanza mediatica.

Proprio la polemica di Pasolini con Moravia a proposito del massacro del Circeo, però, ha la funzione di chiarire le diversità delle posizioni e dell'espressione di entrambi sulla società contemporanea e sui suoi rap- 
porti con la rappresentazione mediatica, ma anche la loro complementarietà. In Le mie proposte per scuola e tv, che segue alla pars destruens delle Lettere Luterane di cui si è detto, Pasolini rimprovera a Moravia di non guardare «le cose stando in mezzo, ma da lontano. Perciò il suo interesse non può riguardare la concretezza o la fisicità» (LL, p. 173). Sempre rifacendosi a Barthes, lo scrittore iniziava il suo articolo dicendo che Moravia "prova il "piacere del testo" solo a patto, come ogni autore del resto, di romanzarlo» (LL, p. I72). Lo scrittore del romanzo Gli Indifferenti aveva infatti paragonato il massacro del Circeo al proprio racconto del 1927 , Delitto al circolo del tennis. Nonostante le indubbie analogie, per Pasolini quest'ultimo rappresentava in realtà un episodio «idillico» rispetto alla verità dei fatti, così come il suo film Accattone (I96I) era idillico rispetto all'analogo episodio dell'aggressione di Cinecittà.

Se per Pasolini il "delitto gratuito "gidiano"» è «diventato un genere di consumo» (pp. I72-I73), per Moravia le stesse coordinate tratteggiano l'impossibilità del tragico nella letteratura contemporanea:

Mi si chiariva insomma l'impossibilità della tragedia in un mondo nel quale i valori non materiali parevano non avere diritto di esistenza e la coscienza morale si era incallita fino al punto in cui gli uomini, muovendosi per solo appetito, tendono sempre più a rassomigliare a degli automi. (UF, p. 48.)

Già nel periodo fascista, inoltre, Moravia individuava una "tristezza» di corporeità e di linguaggio simile a quella sottolineata da Pasolini. Come scriveva in La noia (1960):

Sono nato nel 1920 , la mia adolescenza passò, dunque, sotto l'insegna nera del fascismo, ossia di un regime politico che aveva retto a sistema l'incomunicabilità così del dittatore con le masse come dei singoli cittadini tra di loro e con il dittatore. La noia, che è mancanza di comunicazione con le cose, era nell'aria stessa che si respirava. (N, p. II.)

Spero di non essere andata troppo lontana nella mia analisi, che intendeva mettere in evidenza anche qui una forma di comunicazione distorta, inautentica, irreale e - per così dire - malata, la stessa che Pasolini diagnostica nella società a lui contemporanea. Non solo, ma lo scrittore vede nel «potere consumistico (con la sua pretesa tolleranza)» una degenerazione ancora peggiore della retorica fascista. Eppure, nonostante il diverso approccio con il problema (Moravia dava a Pasolini del «preraffaellita»; quest'altro, come abbiamo visto, accusava l'amico di stare ad eccessiva distanza, di filtrare tutto attraverso il romanzesco, di non avere compreso come i contorni delle cose non fossero più nitidi, ma "due confusi, magmatici, disordinati, irriducibili, sbavanti campioni di una qualità di vita»: LL, p. 174), entrambi, in fondo, arrivano a riconoscere di dire "quasi 
esattamente le stesse cose» (LL, p. I77). Entrambi, in particolare, ravvisano nel conformarsi della società di massa all'ideale consumistico e nella degenerazione del capitalismo - come rovesciamento dei rapporti mezzofine tra uomini e cose, come estensione del concetto simbolico di capitale a tutta la realtà - una forma di distruzione dell'umanità nei suoi aspetti più autentici come il linguaggio, la corporeità, considerati tramite di una corrispondenza libera, piena, felice tra gli uomini. Per Pasolini è ancora più feroce, come abbiamo visto, la critica alla risonanza mediatica di questo fenomeno. Cito ancora gli Scritti corsari, a proposito di questa diseducazione: «il ragazzo piccolo-borghese, nell'adeguarsi al modello "televisivo" che, essendo la sua stessa classe a creare e a volere, gli è sostanzialmente naturale, diviene stranamente rozzo e infelice. Se i sottoproletari si sono imborghesiti, i borghesi si sono sottoproletarizzati» (dall'articolo apparso sul Corriere della Sera il 9 dicembre 1973 con il titolo "Sfida ai dirigenti della televisione», in Scritti corsari intitolato Acculturazione e acculturazione, SC, p. 24). Moravia, è vero, privilegia le metafore per delineare la schiavitù dell'uomo al consumo: basti pensare alla Noia, alla grottesca riscrittura del giovane che si immola al vitello grasso (e non viceversa), alla proiezione della frenesia consumistica nella simbologia sessuale, in questo romanzo e altrove, ma anche alla ricerca di antidoti tratti dal mondo naturale e sempre metaforizzati in un'imagerie molto ricca e diversificata.

Vorrei dunque concludere considerando come i punti di vista dei due scrittori si avvicinino - pur usando talvolta un linguaggio e un immaginario diverso (più esplicito, almeno nelle pagine che vi ho letto, quello di Pasolini; più implicito, simbolico, quello di Moravia) - in due racconti di Moravia come Il diavolo non può salvare il mondo e C’è una bomba $N$ anche per le formiche, tratti da La cosa e altri racconti (1983), in cui i cambiamenti e le aberrazioni che si collegano alle nuove forme di comunicazione (la televisione e i mass media), sono proposti in modo ancora più intenso e problematico grazie all'elaborazione letteraria con cui sono trasmessi. In entrambi i casi, le aporie della società vengono affrontate attraverso il paradosso. Attraverso un'amplificazione parossistica, il consumismo è degenerato nell'incubo nucleare. Nel primo racconto viene riproposto il tema topico del patto con il diavolo, che va da Marlowe a Mann, a Boito, a Valéry, ecc. Qui, come nel rovesciamento parodico del vitello grasso, non è più l'uomo - ormai completamente padrone di sé - a innamorarsi del diavolo, ma è quest'ultimo che si innamora di colui, tale Gualtieri, che rappresenta la saturazione (e dunque la resistenza) dell'uomo di fronte a ogni desiderio. Il diavolo assume tutte le forme e sembra assecondare tutte le perversioni, ma senza successo: soltanto quando assumerà la forma del 
sesso femminile, vero oggetto parziale per eccellenza, riuscirà a farsi possedere, per scomparire poi nel momento culminante del rapporto, lasciando soltanto come traccia di sé un «tenue fumo tremulo, che potrebbe benissimo essere uscito dal motore surriscaldato dell'automobile» (CR, p. IO7). Anche nell'inedito I due amici, scritto nel 1953 e recentemente pubblicato - una sorta di palinsesto con tre redazioni differenti di una medesima storia (si racconta di Sergio, «intellettuale» e "comunista», e di Maurizio, «borghese» ricco e viziato ma carismatico, e molto restio a farsi «convertire» alla fede politica dall'amico) - soprattutto nella seconda redazione tutta la vicenda può essere letta come un patto con il diavolo. Tra i due amici si inserisce il personaggio di Lalla, la compagna di Sergio (che nelle altre due versioni si chiama Nella: appena introdotta nella prima, più remissiva e animalesca nella terza). La ragazza - paragonata a "quei rettili eleganti e goffi delle epoche antidiluviane» ma, insieme, "profondamente attraente» (DA, p. IO6) - diventa oggetto del contendere tra i due amici, perché Maurizio promette che si convertirà al comunismo soltanto se Sergio gli concederà di andare a letto con lei. Il patto diabolico «messo in scena» da Maurizio, una trappola in cui l'«intellettuale» Sergio cade per troppa sicurezza, ha una funzione paradossalmente benefica: smascherare come un ideale politico non possa fare dell'uomo soltanto un mezzo per il raggiungimento del suo fine. La donna diventa per provocazione il simbolo di questo rovesciamento di valori: la mortificazione dell'amore e del corpo consacrati all'ideale e ridotti a merce di scambio e, al tempo stesso, la persuasione politica come mera conquista di voti, di anime vendute al demonio più che di autentiche e ponderate scelte. All'utopia si affianca così contemporaneamente l'antiutopia, con la ribellione della donna che si impone con intelligenza e in tutta la sua fiorente carnalità e si sottrae, alla fine, ai disumanizzanti esperimenti di entrambi.

Soggezione al potere politico, edonismo e consumo come nuove religione: due punti vista che richiamano ancora una volta Pasolini. Un punto di vista che, per l'autore di Le ceneri di Gramsci, non risparmia anche il conformismo di sinistra, con la sua volontà di «sconsacrare e (inventiamo la parola) de-sentimentalizzare la vita» (LL, p. 2I).

Naturalmente tra I due amici, scritto nel 1953 e i racconti di La cosa (1983) sono passati trent'anni e la società italiana non ha fatto che avverare le allarmate profezie «luterane» di Pasolini. In quest'ultima raccolta di racconti moraviani, il diavolo è veramente tra i simboli più ambigui della scrittura moraviana: segno di un'estenuante Wille che si perverte nel consumo, sua spietata rivelazione, figura di una provocatoria apocalisse che indica una via d'uscita (la sottrazione del consumo, del godimento 
ideologico) nel momento in cui mostra, rivela la più grande forza vitale dell'uomo e la sua più tragica perversione. In C’è una bomba $N$ anche per le formiche l'astuzia mediatica è implicitamente richiamata fin dalle prime righe del racconto:

Alle sette del mattino, al mare, dopo avere spalancato la finestra, gli piace buttarsi tutto nudo sul letto, prendere il primo libro o rivista o giornale che gli capita sottomano e leggere per dieci, quindici minuti una cosa qualsiasi, per svegliarsi del tutto, per riprendere contatto con il mondo. Preferibilmente, qualche cosa di catastrofico, forse per equilibrare il senso di profonda tranquillità che emana dalla finestra piena di un cielo ancora freddo e vuoto, con vaghe tracce, qua e là, di rossori aurorali. Stamani tende la mano verso il pavimento, raccoglie a caso il giornale che la sera prima aveva lasciato cadere, vinto dal sonno, e lo spiega. Sì, ci vorrebbe qualche cosa di drammatico, magari di catastrofico. Ecco, su quattro colonne, il titolo che cercava, sul pro e il contro della bomba N. Benissimo, che cosa di più catastrofico della fine del mondo? $\mathrm{Si}$ accomodo meglio il cuscino sotto la testa, porta il giornale all'altezza degli occhi e legge. (CR, p. 235.)

C'è in questo scorcio la morbosa attenzione dell'uomo per la notizia catastrofica, come si dice diffusamente apocalittica, che la divulgazione di stampa e tv tende a sfruttare e ad esaltare in tutta la sua portata spettacolare. Il protagonista è però anche un uomo intelligente e capisce che sia l'attenzione per questi eventi sia la loro origine (l'evidenza di un orrore come la bomba N) deriva dalla premessa che «l'umanità vuole morire». $\mathrm{Ne}$ è spinta dalla sua voglia di vivere, da quella stessa Wille che induce le formiche ad andare in cerca di miele, a spingersi «sul sentiero di guerra» (CR, p. 236) e a farsi uccidere dall'insetticida (quello che è per loro la bomba $\mathrm{N}$, di qui il titolo). L'osservazione della natura, la sua discreta, silenziosa presenza, è ancora una volta per Moravia un antidoto. La curiosità mediatica si fonde inevitabilmente, per il personaggio di questo breve racconto, all'osservazione del comportamento animale, al relativismo che ne deriva:

Ne avrà ammazzate, diciamo, mille. Ma questa strage si è svolta nel silenzio, lui non ha sentito nulla. Eppure, chissà, forse le formiche si lamentavano, gridavano, urlavano. $\mathrm{E}$ ancora chi ha mai visto l'espressione della formica nel momento che muore colpita dall'insetticida? Agli uomini appare un puntino nero, niente di più? (C, p. 238.)

L'interpretazione sociologica dei meccanismi di consumo si apre a una considerazione filosofica e letteraria più ampia, ambigua, polisemica, che va da Leopardi a Shopenhauer, dall'Ecclesiaste (citato) a Camus. Che il pensiero dell'Ecclesiaste sia vero («Nulla di nuovo sotto il sole») è valido fino al 1945, "fino, cioè, alla bomba atomica». Aldilà della sapienza antica e della presunzione umana, ma anche della spettacolarità mediatica, l'uomo dovrebbe arrendersi ancora una volta alla lezione dell'animale: 
L'ultima di queste cose nuove è la bomba N. Puoi forse dire, a proposito della bomba N, niente di nuovo sotto il sole? Eh no, proprio no. E allora, forse, delle cose di cui non si può parlare, è meglio tacere. (CR, p. 239.)

Le formiche, le lucciole (siamo di nuovo a Pasolini, «L'articolo delle lucciole», I febbraio 1975, "darei l'intera Montedison per una lucciola»...) insegnano a tacere e, con buona pace di Pasolini, anche a spegnere la televisione. Nella convergenza di questa immagine, quindi, possiamo cogliere non solo la critica di questi due scrittori all'azione dei mass media nella società del tempo, ma anche il senso del loro profondo, rinnovato umanesimo.

\section{Bibliografia}

\section{Opere di Pierpaolo Pasolini}

LL Lettere luterane. Il progresso come falso progresso, Torino, Einaudi, 2003 (1976).

SC Scritti corsari, Milano, Garzanti, 1975.

\section{Opere di Alberto Moravia}

DA I due amici, a cura di S. Casini, Milano, Bompiani, 2007.

UF L'uomo come fine e altri saggi, Milano, Bompiani, I972 (I963).

N La noia, Milano, Bompiani, I960.

CR La cosa e altri racconti, Milano, Bompiani, 1983.

\section{Altri testi citati}

BARThes Roland, Variazioni sulla scrittura seguite da Il piacere del testo, Torino, Einaudi, 1999 [or. Le plaisir du texte, 1973].

ŽıžEx Slavoj, In Defense of Lost Causes, London, Verso, 2008.

ŽIžEK Slavoj e DALY Glyn, Psicoanalisi e mondo contemporaneo, Bari, Dedalo, 2006 [or. Conversations with Žižek].

ŽıžEK Slavoj, Il soggetto scabroso: trattato di ontologia politica, Milano, Cortina, 2003 [or. The ticklish subject: the absent centre of political onto$\log y$, 1999].

ŽıžEK Slavoj, Il godimento come fattore politico, Milano, Cortina, 200I [or. For they know not what they do: Enjoyment as a political factor, I99I]. 
